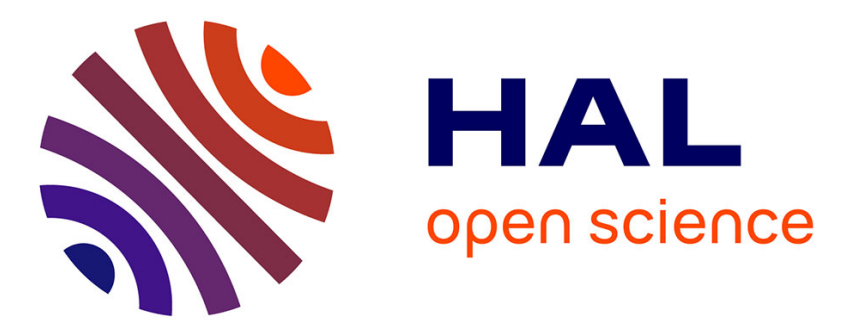

\title{
Anisotropy of Elastic and Relaxation Properties of the Superconducting 123-YBCO Single Crystal
}

\author{
P. Pal-Val, L. Pal-Val, V. Demirsky, V. Natsik, M. Sorin
}

\section{To cite this version:}

P. Pal-Val, L. Pal-Val, V. Demirsky, V. Natsik, M. Sorin. Anisotropy of Elastic and Relaxation Properties of the Superconducting 123-YBCO Single Crystal. Journal de Physique IV Proceedings, 1996, 06 (C8), pp.C8-489-C8-492. 10.1051/jp4:19968106 . jpa-00254534

\section{HAL Id: jpa-00254534 https://hal.science/jpa-00254534}

Submitted on 1 Jan 1996

HAL is a multi-disciplinary open access archive for the deposit and dissemination of scientific research documents, whether they are published or not. The documents may come from teaching and research institutions in France or abroad, or from public or private research centers.
L'archive ouverte pluridisciplinaire HAL, est destinée au dépôt et à la diffusion de documents scientifiques de niveau recherche, publiés ou non, émanant des établissements d'enseignement et de recherche français ou étrangers, des laboratoires publics ou privés. 


\title{
Anisotropy of Elastic and Relaxation Properties of the Superconducting 123-YBCO Single Crystal
}

\author{
P.P. Pal-Val, L.N. Pal-Val, V.V. Demirsky, V.D. Natsik and M.N. Sorin \\ B. Verkin Institute for Low-Temperature Physics \& Engineering, National Academy of Sciences of \\ Ukraine, 47 Lenin Ave., 310164 Kharkov, Ukraine
}

\begin{abstract}
Acoustic properties of the $\mathrm{YBa}_{2} \mathrm{Cu}_{3} \mathrm{O}_{6.7}$ single rystal are studied in the temperature range $5-300 \mathrm{~K}$. The wave vector of the longitudinal and torsional standing waves in the frequency range $50-90 \mathrm{kHz}$ was directed along and normal to the $c$-axis. It is shown that the acoustic anomalies observed in the single crystal qualitatively are the same that those found earlier in the 123-YBCO ceramics but depend strongly on the mutual orientation of the wave vector and the $c$-axis. I1 is established that the significant temperature hysteresis and "softening" of the dynamic elastic moduli at thermocycling are observed only in the case when the sound waves have a displacement component along the $c$-axis. Moreover, in this case the sound absorption peak near $230 \mathrm{~K}$ is more pronounced. The locations of sound absorption peaks are not sensitive to the oscillation mode and sample orientation. The results obtained are qualitatively consistent with the model of bistable behaviour of the sublattice formed by apical oxygen atoms.
\end{abstract}

\section{INTRODUCTION}

A number of well reproduced anomalous effects was found when studying temperature dependences of acoustic properties of $\mathrm{YBa}_{2} \mathrm{Cu}_{3} \mathrm{O}_{\mathrm{x}}$ ceramics in the temperature range $5-300 \mathrm{~K}$. Sound absorption peaks (number of which may run up to 5) and significant hysteresis of elastic moduli at thermocycling were the most prominent among them (see, for example, $[1,2]$ ).

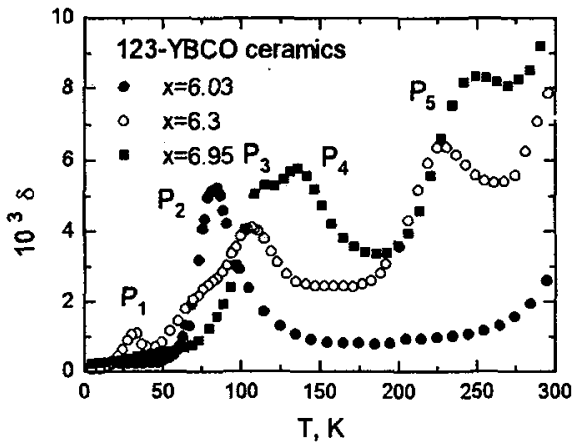

Figure1: Temperature dependences of decrement in 123YBCO ceramics with different oxygen content.

The number and intensity of the absorption peaks depend to a great extent on the oxygen content. Fig. 1 shows the temperature spectra of internal friction obtained by us earlier in the ceramics samples with different $x[3-5]$. It is seen that the peak $P_{2}$ is present only in the tetrahonal phase at $x \rightarrow 6$ (see also [6]). The peak $\boldsymbol{P}_{3}$ is observed both in the orthorhombic $(x>6.5)$ and tetrahonal phases. The peak $P_{4}$ occurs only in the orthorhombic phase and its height goes down when the degree of orthorhombicity reduces [4]. The peaks $P_{1}$ and $P_{5}$ might be observed in all the modifications of the ceramics $(6.03<x<6.95)$, but their intensity and position along the temperature axis vary in a nonregular way and seem likely to depend on technology factors. It is established that the peaks $P_{2}, P_{3}$ and $P_{4}$ are due to thermoactivated relaxation processes and values of the activation energies $U_{P_{2}}=0.101 \mathrm{eV}, U_{P_{3}}=0.16 \mathrm{eV}, U_{P_{4}}=0.19$ $\mathrm{eV}$ and the attack frequencies $v_{P_{2}}=1.1 \cdot 10^{12} \mathrm{~s}^{-1}, v_{P_{3}}=6 \cdot 10^{12} \mathrm{~s}^{-1}, v_{P_{4}}=9 \cdot 10^{12} \mathrm{~s}^{-1}$ are obtained. The peaks $P_{1}$ and $P_{5}$ are rather due to phase transformations of some kind [1,7]. 
The temperature boundaries and magnitude of the elastic moduli hysteresis vary within wide limits in different experiments [1]. The typical temperature range of the hysteresis is $50-220 \mathrm{~K}$, the maximum magnitude of the hysteresis varies from total absence up to $20-30 \%$ of values measured [8].

Inspite of a great number of works, satisfactory explanations of the effects observed are absent. In part, it may be because the researchers are unable to answer the question whether a certain acoustic anomaly in ceramics is connected with the process in grains or is localized at grain boundaries. The only way to answer this question is obtaining the experimental data on single crystals. A number of works dealing with single crystals is much less than that for ceramics [9-12]. Single crystals studied had a shape of thin plates and therefore it was difficult to carry measurements along $c$-axis. Besides an influence of a crystal surfaces was considerable.

In this work, a study of the temperature dependences of sound absorption and relative changes of elastic moduli was carried out in the range $5-300 \mathrm{~K}$ for a bulk 123-YBCO single crystal with excitation of various vibration modes along different crystallographic directions in the sample.

\section{EXPERIMENTAL}

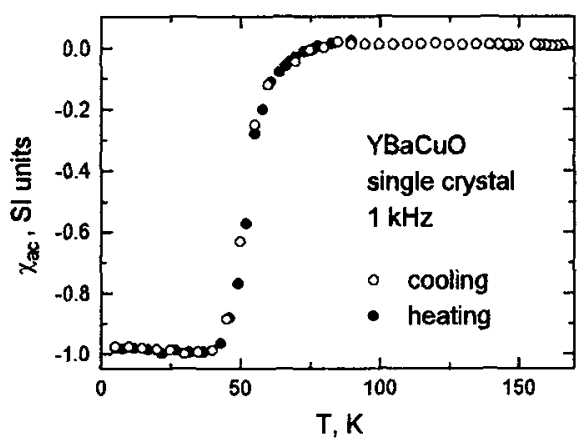

Figure 2. Temperature dependences of ac-magnetic susceptibility in the sample studied.

Table 1.

\begin{tabular}{|c|c|c|c|}
\hline №/№ & Vibration mode & $q$ & $u$ \\
\hline 1 & longitudinal & $q \| c$ & $u \| c$ \\
\hline 2 & longitudinal & $q \perp c$ & $u \perp c$ \\
\hline 3 & torsional & $q \| c$ & $u \perp c$ \\
\hline 4 & torsional & $q \perp c$ & $u \| c$ \\
\hline
\end{tabular}

The YBCO-single crystal with dimensions $5 \times 2.6 \times 2.2 \mathrm{~mm}^{3}$ was grown from YBCO solution in eutectic mixture of $0.28 \mathrm{BaO}+0.72$ $\mathrm{CuO}$. The solution was heated on air in zirconia crucible up to $1010^{\circ} \mathrm{C}$, then was kept at this temperature during 10-12 hours and finally was cooled with the rate $\sim 0.06 \mathrm{~K} /$ hour. Superconducting properties were investigated by measuring the temperature dependences of ac-magnetic susceptibility $\chi_{a c}$ at the frequency $1 \mathrm{kHz}$ and at the magnetic field amplitude of $0.1 \mathrm{mT}$ (Fig.2). The temperature of the onset of the superconducting transition $T_{c o}=75 \mathrm{~K}$. According to [13] it corresponds approximately to $x=6.7$.

The acoustic measurements were made by the two-component oscillator technique at $88 \mathrm{kHz}$ in the range $5-300 \mathrm{~K}$. In order to put the sample into the wave antinode, it was glued between two buffer quartz rods. The longitudinal and torsional standing waves with the amplitude $\varepsilon_{0} \cong 2 \cdot 10^{-7}$ were excited in the sample. Four different cases of mutual orientations of the wave vector $\boldsymbol{q}$, the displacement vector $\boldsymbol{u}$ and the $c$-axis were realized in the experiment (Table 1). In view of a presence of twins, we did not control the wave vector direction in the plane $a b$.

\section{RESULTS}

Fig. 3 presents the temperature dependences of the decrement $\delta(T)$ measured during cooling and subsequent heating. It is seen that for the chosen mutual orientations of $q, u$ and $c$-axis only the peaks $P_{l}, P_{4}$ and $P_{5}$ are observed in the single crystal. Besides, the peaks $P_{1}$ and $P_{5}$ seem to be more pronounced when the sound wave has a displacement component along the $c$-axis.

Fig. 4 shows the temperature dependences of the relative variation of the elastic moduli $\Delta E / E(T)$ and $\Delta G / G(T)$ measured during cooling and heating. One of the main features of these dependences is a wide temperature hysteresis of the elastic properties at thermocycling. The hysteresis has a maximum value in the case when ultrasonic wave has a displacement component along $c$ - axis. In this case another important peculiarity also manifests itself, viz. significant "softening" 

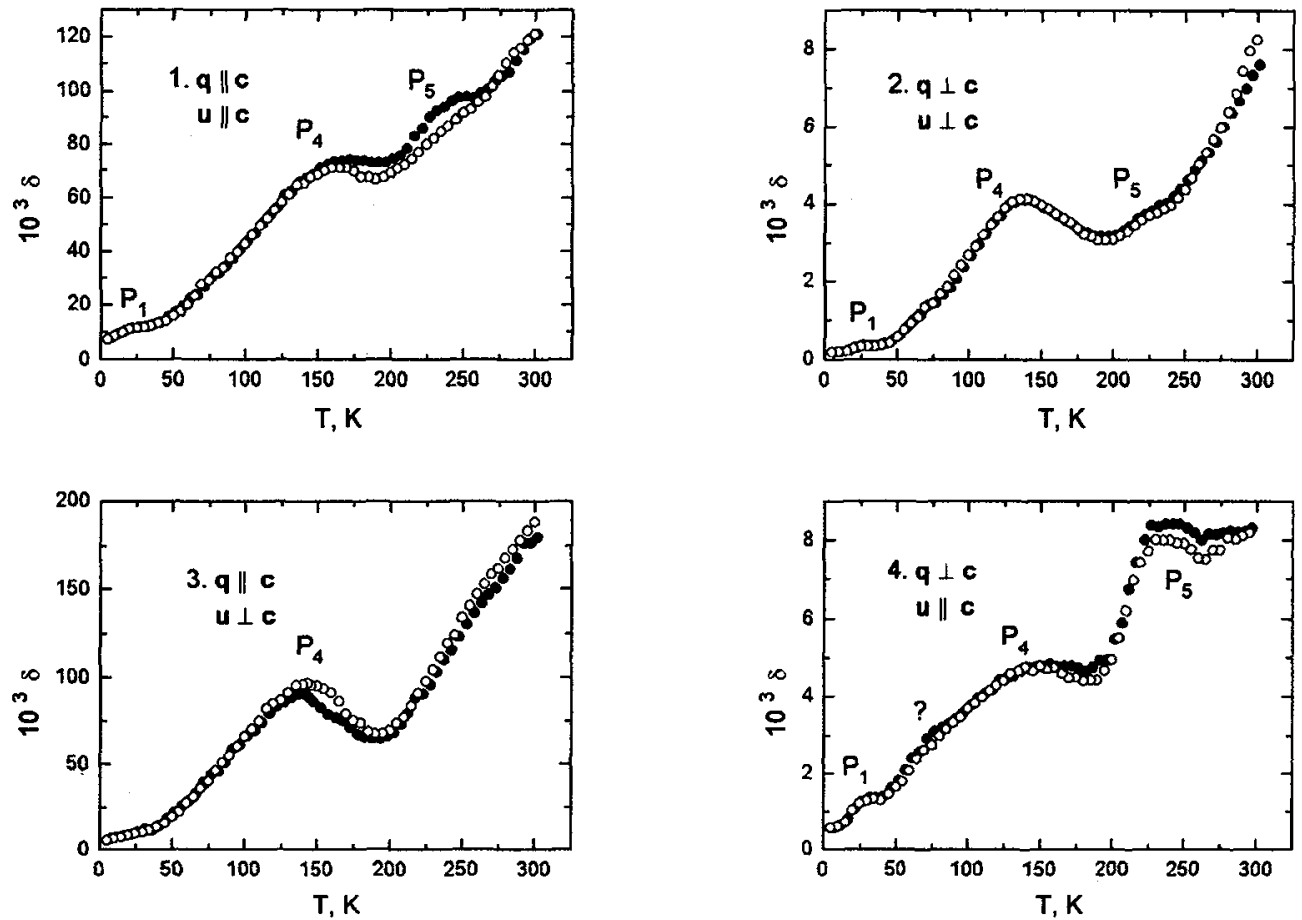

Figure 3. Temperature dependences of the decrement for different mutual orientations of the wave vector $q$, displacement vector $u$ and $c$-axis (see Table 1 ).

of the corresponding elastic moduli below $\sim 200 \mathrm{~K}$. Up to now, the data on hysteresis of elastic properties of 123-YBCO single crystal were only reported in [12]. Results of [12], obtained in the $\mathrm{MHz}-$ frequency range, are consistent qualitatively with our data.

\section{DISCUSSION AND CONCLUSIONS}

The experimental data obtained in this work allow us to make some remarks on the nature of acoustic anomalies observed in the 123-YBCO composition. First of all, it should be noted that the most of the effects observed before in the 123-YBCO ceramics in the range $5-300 \mathrm{~K}$ are present also in the single crystals. Therefore it may be concluded that at least the sound absorption peaks $P_{1}, P_{4}$ and $P_{5}$ as well as the hysteresis and significant "softening" of the elastic moduli at thermocycling are due to processes occuring in the volume of crystals rather than at the grain boundaries. An absence of the peak $P_{2}$ can be explained by the comparatively high value of the oxygen index. But the lack of the peak $P_{3}$, that usually looks like a low-temperature component of the composite peak $P_{3}-P_{4}$ in ceramics, seems rather surprising and may indicate that the corresponding relaxation process cannot take place at the chosen mutual orientations of the wave-vector $q$, displacement vector $\boldsymbol{u}$ and crystallographic axes.

The results obtained show that the hysteresis of the elastic properties at thermocycling cannot be explained by twin motion because the hysteresis has the maximum value when the displacement vector $u$ lies in twin boundary planes $\{110\}$. The fact that the essential temperature hysteresis and strong "softening" of the elastic moduli are observed only when the component of displacement vector along $c$-axis is present may count in favour of the model suggested in [14] according to which the possible reason of the elastic moduli hysteresis in 123-YBCO may be the bistable behaviour of the sublattice of apical oxygen $O(4)$.

To achieve a further progress in understanding of the nature of the anomalies observed it is 

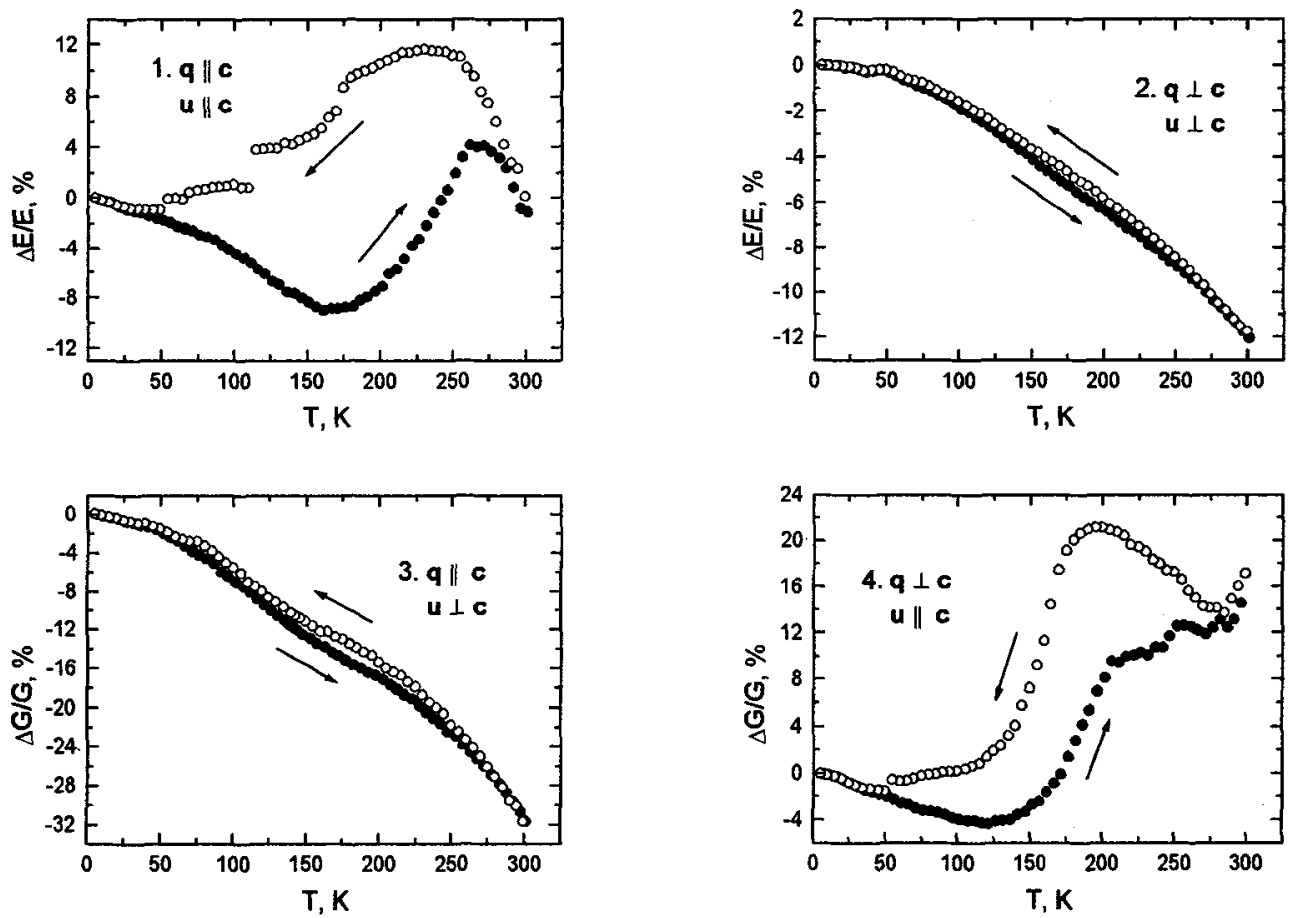

Figure 4. Temperature dependences of the elastic moduli for different mutual orientations of the wave vector $q$, displacement vector $u$ and $c$-axis (see Table 1 ).

necessary to perform investigations on the single crystals with different oxygen content and controlled twin structure.

\section{REFERENCES}

[1] Natsik V.D. and Pal-Val P.P., Sov.J.Low Temp.Phys. 16 (1990) 475-476.

[2] Gzowski O., Davoli I., Stizza S.,Kusz B., Barczynski R., Gazda M. and Murawski L. Rev. Solid State Science 5 (1991) 43-52.

[3] Natsik V.D., Pal-Val P.P., Engert J., Kaufmann H.-J., Rudolph K. and Matz W., Sov.J.Low Temp.Phys. 16 (1990) 160-163.

[4] Pal-Val P.P., Pal-Val L.N., Demirskii V.V., Natsik V.D. and Prytkin V.V., Superconductivity 4 (1991) 1450-1459.

[5] Pal-Val L.N., Pal-Val P.P., Natsik V.D. and Dotsenko V.I.,Solid State Commun.81 (1992) 761 765.

[6] Cannelli G., Cantelli R. and Cordero F. Phys. Rev. B 38 (1988) 7200-7205.

[7] Pal-Val L.N., Pal-Val P.P., Natsik V.D. and Dotsenko V.I., Sov.J.Low Temp.Phys. 18 (1992) 86-92.

[8] Pal-Val P.P., Natsik V.D., Engert J., Kaufmann H.-J., Rudolph K. and Matz W., Cryst. Res. Technol. 24 (1989) 1151-1158.

[9] Hoen S., Bourne L.C., Kim C.M. and Zettl A., Phys. Rev. B 38 (1988) 11949-11951.

[10] Saint-Paul M. and Henry J.Y., Solid State Commun. 72 (1989) 685-687.

[11] Shi X.D., Yu R.C., Wang Z.Z., Ong N.P. and Chaikin P.M., Phys. Rev. B 39 (1989) 827-830.

[12] Kim T.J., Kowalewski J., Assmus W. and Grill W., Z.Phys.B: Cond. Matter 78 (1990) 207-212.

[13] Cava R.J. Science 247 (1990) 656-662.

[14] Saiko A.P., Gusakov V.E. and Kuz'min V.S., Physica C 235-240 (1994) 1239-1240. 